\title{
Evidencia científica de la relación entre acoso laboral y depresión
}

\section{Evidence based relationship between mobbing and depression}

\section{Víctor Adrián Harasemiuc ' ${ }^{\text {, Jesús Raúl Díaz Bernal }}{ }^{2}$}

1 Unidad Docente de Medicina del Trabajo de la Comunidad de Madrid, Madrid 1: Escuela Nacional de Medicina del Trabajo- Instituto de Salud Carlos III, Hospital de la Princesa. Madrid. España.

2 Unidad Docente de Medicina del Trabajo de la Comunidad de Madrid, Madrid 1: Escuela Nacional de Medicina del Trabajo- Instituto de Salud Carlos III, IBERMUTUAMUR. Madrid. España.

Recibido: 20-05-13

Aceptado: 08-08-13

\section{Correspondencia}

Víctor Adrián Harasemiuc

Teléfono: +34 656543823

Correo electrónico: haradrian@gmail.com

Resumen

Introducción: El Acoso laboral es una epidemia silenciosa que no solamente afecta a la motivación y a la productividad de los trabajadores, sino que también, afecta negativamente a su víctimas en aspectos psicosociales siendo la depresión una de sus consecuencias.

Objetivos: El objetivo es identificar la evidencia científica conocida sobre la relación existente entre el acoso laboral y la depresión.

Metodología: Se formularon una serie de ecuaciones de búsqueda con los términos: Mobbing, Depression, Workplace Bullying, Harassment, Depresión y Acoso Laboral, que se aplicaron a diferentes bases de datos bibliográficas (IBECS, LILACS, The Cochrane Library Plus, Scielo, WHOLIS, OSH Update), que permitió la identificación de 36 referencias de las cuales 8 cumplieron los criterios de inclusión. En función de los objetivos planteados se extrajo la información respectiva después de su revisión a texto completo.

Resultados: La prevalencia de acoso laboral fluctuó entre un 11,9\% y un 81\% según los países en los que se realizó el estudio. Ser mujer, tener estudios universitarios y la antigüedad o experiencia en el puesto de trabajo son factores que determinaron una mayor vulnerabilidad para sufrir acoso. El perfil del acosador es: ser de sexo masculino y, generalmente, de categoría superior a la víctima; siendo el abuso verbal, el hostigamiento y el aumento de la carga laboral las estrategias de acoso más utilizadas. En todos los estudios se encontró una relación positiva entre el acoso y la depresión.

Conclusiones: La revisión realizada, no permite demostrar una relación de causalidad entre el acoso y depresión. La reproducibilidad en los resultados de los estudios evidencian la existencia de una asociación entre acoso y depresión, siendo necesario el promover estudios de diseño longitudinal que puedan demostrar, o no, una asociación causal.

Med Segur Trab (Internet) 2013; 59 (232) 361-371

Palabras Clave: Acoso laboral, depresión, riesgo psicosocial. 
Abstract

Introduction: Workplace bullying is a silent epidemic that affects motivation and productivity of workers, but there are also consequences in psychosocial level being depression one of its.

Objectives: The objective is to identify the scientific evidence on the relationship between bullying and depression.

Methods: We formulated a set of equations search using the terms: Mobbing, Depression, Workplace Bullying, Harassment, Workplace Bullying Depression, which were applied to different bibliographic databases (IBECS, LILACS, The Cochrane Library Plus, SciELO, WHOLIS , OSH Update), which allowed the identification of 36 references of which 8 met the inclusion criteria. In relation with each objectives the information was extracted after full-text review.

Results: The prevalence of bullying ranged between $11.9 \%$ and $81 \%$ depending on the country in which the study was conducted. Female, having a college education and seniority or experience in the job are factors that determined an increased vulnerability to harassment. The profile of the harasser is: male, and generally superior to the victim, verbal abuse, harassment and increased workload are the bullying strategies most used. All studies evidence a positive relationship between bullying and depression.

Conclusions: The review, does not establish a causal relationship between bullying and depression. The reproducibility of the results of the studies show the existence of an association between bullying and depression, being necessary to promote longitudinal studies that could show, or not, a causal association.

Med Segur Trab (Internet) 2013; 59 (232) 361-371

Keywords: Workplace Bullying, depression, psychosocial risk. 


\section{INTRODUCCIÓN}

El Acoso laboral en las últimas décadas ha pasado de ser un tema poco mencionado en la literatura; a convertirse en un problema de importancia creciente; más aún en momentos en que la crisis económica global repercute directa o indirectamente en las condiciones de trabajo y en las relaciones interpersonales entre los trabajadores. Ejemplo de esto se recoge en nuestro país, en la VII Encuesta Nacional sobre Condiciones de Trabajo $^{1}$, que nos muestra que un $11 \%$ de la población trabajadora declara haber sido víctima de alguna conducta violenta en el trabajo, de éstos, un $22,7 \%$ ha contestado "a menudo" y el 84,5\% "a veces"; a nivel Europeo ${ }^{2}$ un $4 \%$ de los trabajadores considera haber sido víctima de acoso laboral, un $2 \%$ de violencia física y un $1 \%$ de acoso sexual.

Los datos anteriores ponen de manifiesto la importancia de conceptualizar, definir y reconocer el acoso laboral; y a su vez conocer sus consecuencias tanto en la salud física como mental de sus víctimas.

El término Acoso Laboral proviene de la traducción del anglosajón Mobbing ${ }^{3}$, término que fue introducido por el etólogo Konrad Lorenz como resultado de sus observaciones de animales en libertad. Usó este término para hacer referencia a los ataques de un grupo de animales pequeños a un animal más grande con el propósito de aterrorizarlo, o para denominar el comportamiento agresivo de un grupo de animales con el objeto de echar a un intruso de su territorio. Posteriormente las ciencias sociales adoptaron este concepto para referirse a ciertas conductas de violencia en el ámbito laboral.

Fue el psicólogo Heinz Leymann quien utilizó el término para referirse a "una situación de terror psicológico en el trabajo que implica una comunicación hostil y amoral, dirigida de manera sistemática por una o varias personas“. En España el Instituto Nacional de Seguridad e Higiene en el Trabajo (INSHT), en su Nota Técnica Preventiva $476^{4}$, se refiere al acoso psicológico o mobbing como "una situación en la que una persona o grupo de personas ejerce una violencia psicológica extrema de forma sistemática y recurrente (como media una vez por semana), durante un periodo prolongado de tiempo (al menos durante seis meses) sobre otra persona o grupo de personas en el lugar de trabajo con la finalidad de destruir las redes de comunicación de la víctima, destruir su reputación, perturbar el ejercicio de sus labores y lograr que finalmente esa persona acabe abandonando el lugar de trabajo“. Más recientemente, el Acuerdo Europeo sobre Acoso y Violencia en el trabajo $(2007)^{3}$, define acoso y violencia como "la expresión de comportamientos inaceptables adoptados por una o más personas, que pueden tomar muy diversas formas, unas más fácilmente identificables que otras". "Se da acoso cuando se maltrata a uno o más trabajadores o directivos varias veces y deliberadamente, se les amenaza o se les humilla en situaciones vinculada con el trabajo".

El acoso laboral tiene un origen multifactorial y depende de diversos factores de riesgo, tanto organizativos como individuales ${ }^{5}$. Por ejemplo desde el punto de vista organizativo un medio laboral que provee a sus empleados un escaso control, una baja capacidad de decisión, un trato poco respetuoso o un estilo autoritario de dirección promueven las conductas violentas; a su vez los factores individuales en los cuales podemos incluir los cognitivos, psicopatológicos y de personalidad también influyen directa o indirectamente en el origen y mantenimiento de estas conductas.

Para entender el problema es importante conocer e identificar las Acciones de acoso $^{3}$, las cuales son el medio por el cual el acosador actúa; podemos agruparlas en: acciones que van dirigidas contra la reputación y dignidad de la víctima, acciones contra el ejercicio de su trabajo, acciones que manipulan la información y la comunicación, y acciones de injusticia laboral. También podemos identificar Conductas de acoso como son los ataques a las víctimas con medidas organizacionales, ataques a las relaciones sociales de la víctima que conllevan aislamiento social, ataques contra la vida privada, ataques a las actitudes de la víctima, agresiones verbales, y violencia física que incluye la sexual. 
La VII Encuesta Nacional sobre Condiciones de Trabajo $^{1}$, nos muestra que las agresiones verbales, rumores o aislamiento social representan son las conductas violentas más frecuentes con un 7,3\%, seguido de amenazas de violencia física con un 3,8\% y de violencia física cometida por personas no pertenecientes a su lugar de trabajo con un 2,4\%. A nivel Europeo ${ }^{2}$ el Abuso Verbal representa un 11\%, las Actitudes Humillantes o Amenazas un 5\% y el Acoso sexual un 2\%.

Podemos clasificar el acoso en varias modalidades ${ }^{3}$ pero atendiendo a los sujetos responsables de hostigamiento, diferenciamos entre:

- Acoso moral vertical: Entre diferentes posiciones jerárquicas; este a su vez puede ser descendente (bossing) o ascendente.

- Acoso moral Horizontal: Entre personas de la misma jerarquía.

- Acoso discriminativo: Este tipo de acoso afecta a lo establecido por el Art. 14 de la Constitución Española, incluye el acoso por razón de género o por cualquier otra causa discriminatoria (raza, edad, creencias, orientación sexual...)

- Acoso por motivos de dominación: Esto implica el ejercicio de un poder de dominio sobre las víctimas, para humillarlas, estando regulado por el Art. 15 de la Constitución Española.

- Acoso institucional: Es el llamado acoso por razones organizativas.

Las consecuencias del acoso laboral tienen una repercusión global, tanto para el trabajador, para la organización, para la familia y entorno social, y para la sociedad en su conjunto.

Centrándonos en la víctima ésta puede tener consecuencias físicas (trastornos funcionales, dolores diversos), psíquicas (ansiedad, baja autoestima, frustración, deterioro cognitivo y depresión) y sociales o relacionales (agresividad, hostilidad, inadaptación, intolerancia a las críticas, desconfianza, retraimiento).

El efecto psicológico de la violencia laboral es una de sus consecuencias más habituales, siendo éste una causa creciente de depresión en la población laboral mundial, de allí la importancia de establecer una causalidad, un diagnóstico y un tratamiento adecuado a este problema de salud que en general se ha convertido en una causa importante de absentismo laboral independientemente de su origen laboral o personal.

La depresión se define como un trastorno mental caracterizado por la presencia de tristeza, pérdida de interés o placer, sentimientos de culpa o falta de autoestima, trastornos del sueño o del apetito, sensación de cansancio y falta de concentración.

La palabra "depresión" proviene del término en latín "depressio" que a su vez procede de depressus" que se traduce como "abatido" o "derribado"

Según la Organización Mundial de la Salud (OMS) en el mundo hay más de 350 millones de personas con depresión, un trastorno mental que altera sus vidas. Sin embargo, debido a la estigmatización que todavía existe de este trastorno, muchos de los afectados no reconocen su enfermedad y no buscan tratamiento.

Un estudio reciente respaldado por la OMS reveló que alrededor del 5\% de las personas residentes en la comunidad han padecido depresión durante el último año ${ }^{8}$.

Por ser una enfermedad de ámbito global con una prevalencia cada vez más elevada la OMS inició un programa para prestar asistencia a los gobiernos para que incluyan el tratamiento de la depresión en sus paquetes básicos de atención sanitaria.

Según la VII Encuesta Nacional de Condiciones de Trabajo la depresión es señalada como una enfermedad agravada o producida por el trabajo en un $60 \%$ de los encuestados y $61,1 \%$ de estos trabajadores reconoce su depresión y buscan apoyo y tratamiento ${ }^{1}$.

La depresión puede llegar a hacerse crónica o recurrente y dificultar sensiblemente el desempeño en el trabajo y la capacidad para afrontar la vida diaria. En su forma más 
grave, puede conducir al suicidio ${ }^{7}$. France Télécom $2011^{6}$, si es leve, se puede tratar sin necesidad de medicamentos, pero cuando tiene carácter moderado o grave se pueden necesitar medicamentos y psicoterapia profesional ${ }^{7}$.

Dado el análisis del estado actual del tema el presente trabajo de revisión se dirige a responder los objetivos que mencionamos a continuación.

\section{OBJETIVOS}

El objetivo de esta revisión es identificar la evidencia científica existente sobre la relación entre acoso laboral y depresión.

Son objetivos secundarios:

- Establecer los factores de incrementan la vulnerabilidad al acoso laboral.

- Determinar la existencia de diferencias, entre las poblaciones laborales estudiadas, en cuanto a la prevalencia de acoso laboral.

- Valorar si la depresión como enfermedad agravada o producida por el acoso laboral afecta los niveles de absentismo laboral.

- Objetivar si el género y la actividad económica son condicionantes que determinen alguna diferencia en cuanto al riesgo de sufrir acoso y depresión.

\section{MATERIALES Y MÉTODOS}

Para la consecución de los objetivos de esta revisión se realizó una búsqueda bibliográfica en las bases de datos que se reflejan en la Tabla 1.

Tabla 1. Bases de datos consultadas

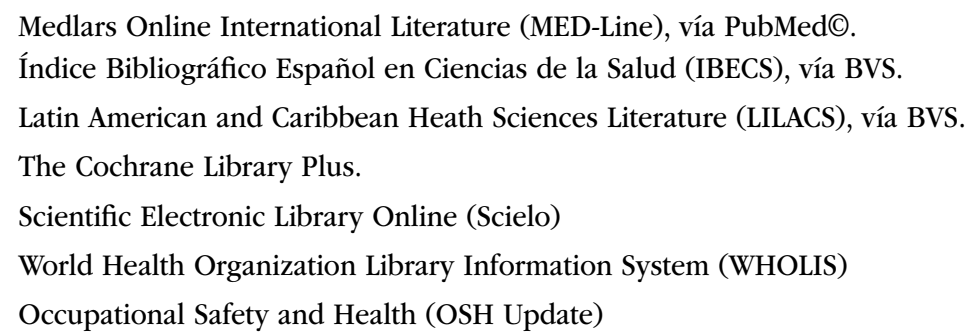

La búsqueda bibliográfica se realizó con un límite temporal, comprendido entre el 1 de enero de 2006 hasta el 14 de noviembre del 2012, utilizando los descriptores y las fórmulas de búsqueda que se reflejan en la Tabla 2.

Tabla 2. Descriptores y fórmulas

\begin{tabular}{ll}
\hline \multicolumn{1}{c}{ Descriptores } & \multicolumn{1}{c}{ Fórmulas de búsqueda } \\
\hline Mobbing & "Mobbing" AND "Depression" \\
Depression & "Acosolaboral" and "depresión" \\
AcosoLaboral & "Workplace Bullying" AND "depression" \\
Workplace Bullying & "Harassment" and "mobbing" \\
Harassment & \\
Depresión & \\
\hline
\end{tabular}


Después de la búsqueda, y tras una evaluación de pertinencia, en base a la lectura de los abstracts, se aplicaron los siguientes criterios de inclusión:

- Estudios que analizan la asociación entre el acoso laboral y depresión.

- Artículos de cualquier diseño metodológico.

- Artículos con abstract en inglés o castellano, que siguieran las pautas de International Committee of Medical JournalEditors (ICMJE).

- Estudios que traten exclusivamente de acoso en el ámbito laboral.

Para recuperar la información de cada artículo se diseñó una tabla en la que extrajo los contenidos de las variables reflejadas en la Tabla 3.

Tabla 3. Información extraída de cada artículo

\begin{tabular}{ll}
\hline Título & Autor y año de publicación \\
Población de estudio & Tamaño de la muestra \\
Diseño del estudio & Variables de exposición \\
Variables efecto & Sesgos \\
Objetivos & Resultados \\
Conclusiones & \\
\hline
\end{tabular}

La selección y revisión de los artículos se realizó mediante la asignación de la mitad de las referencias y artículos seleccionados a cada uno de los autores. La determinación del nivel de evidencia se realizó de acuerdo a los criterios SIGN.

\section{RESULTADOS}

De la aplicación de las fórmulas de búsqueda, se recuperaron 36 referencias cuya distribución por bases de datos figura en la Tabla 4. Tras eliminar los artículos duplicados y hacer una lectura de pertinencia de los abstracts, se seleccionaron 20 referencias.

Una vez aplicados los criterios de inclusión se seleccionaron 8 artículos para su inclusión en el análisis y revisión a texto completo (Figura 1): 6 estudios transversales y 2 estudios prospectivos; 3 estudios en población Turca, 2 estudios en población Francesa, 2 estudios en población Japonesa y 1 estudio en población Danesa.

Tabla 4. Flujo de información en función de las bases de datos consultadas

\begin{tabular}{lcc}
\hline \multicolumn{1}{c}{ Base de datos } & $\begin{array}{c}\mathbf{N}^{\circ}{ }^{\circ} \text { de referencias } \\
\text { encontradas }\end{array}$ & $\begin{array}{c}\mathbf{N}^{\circ} \text { total }_{\text {artículos }} \\
\text { seleccionados }\end{array}$ \\
\hline Medline viaPubMed & 15 & 7 \\
IBECS vía BVS & 13 & 1 \\
LILACS viaBVS & 0 & 0 \\
The Cochrane Library Plus & 0 & 0 \\
Scielo & 2 & 0 \\
WHOLIS & 3 & 0 \\
OSHUpdate & 3 & 0 \\
\hline
\end{tabular}


Figura 1. Diagrama de flujo de la selección de artículos

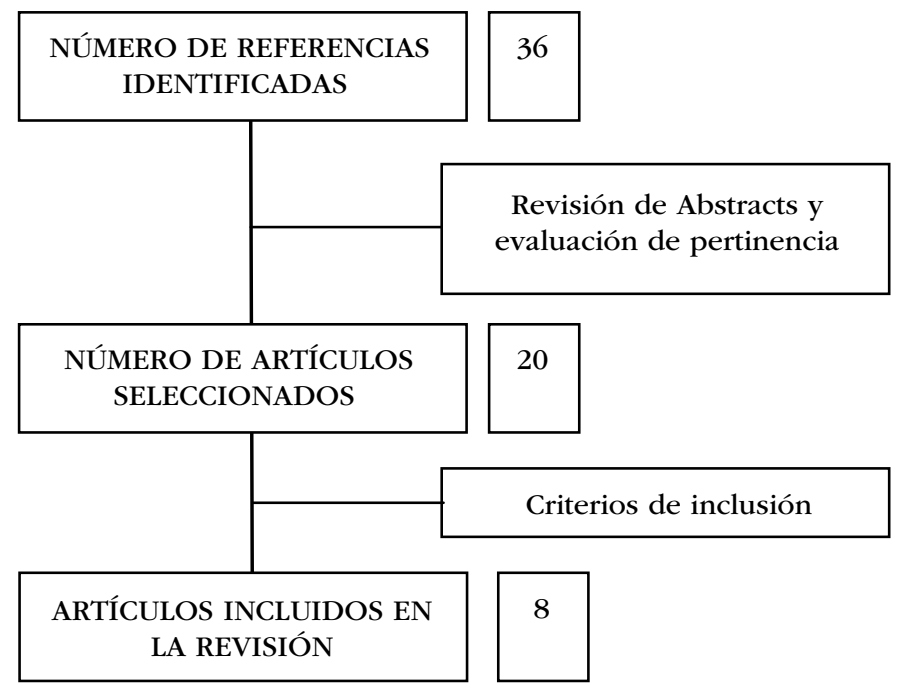

En respuesta al objetivo principal relativo a la relación entre acoso y depresión Rugulies $\mathrm{R}^{16}$ en 2012 en un estudio prospectivo con una muestra de 5.701 cuidadoras de ancianos, observó un incremento de la frecuencia de cuadros de depresión en trabajadoras que referían situaciones de acoso; a las trabajadoras se les evaluó en dos momentos con un intervalo de 20 meses; encontrando una relación entre el acoso laboral y la depresión con una OR de 6,29 (IC 95\% 2,25 - 15,68) en trabajadoras que ocasionalmente habían sido víctimas de acoso y una OR de 20,96 (IC 95\% 5,80 - 75, 80) en trabajadoras que de forma frecuente habían sufrido situaciones de acoso.

Taniguchi T. ${ }^{15}$ en 2011 en un estudio transversal con una muestra de 897 cuidadoras de ancianos, encontró que la prevalencia de la depresión fue significativamente mayor en las cuidadoras expuestas que las no expuestas a acoso $(\mathrm{p}<0,05)$.

También con un diseño transversal Yildirim D. ${ }^{14}$ en 2009 estudia a un colectivo de 286 enfermeras de un hospital de Ankara, encontrando que un 45\% de las encuestadas presentaban síntomas de depresión moderada o grave, y de éstas un 33\% habían sido víctimas de acoso, observándose una correlación positiva y significativa entre la depresión y el acoso $(\mathrm{P}<0,00)$.

Niedhammer ${ }^{13}$ estudia, en 2006, mediante un diseño transversal una muestra de 7.692 trabajadores Franceses, concluye en la existencia de una asociación, entre el acoso laboral y los síntomas depresivos, relación que es independiente del género; resultados en esa misma dirección los refiere Serpil Aytac ${ }^{9}$ en 2011 con una muestra de 1.708 trabajadores.

En 2010 JiroTakaki $^{10}$ con el objetivo de encontrar la existencia de una asociación entre acoso laboral y depresión, realizó un estudio transversal con 2.634 trabajadores de distintas empresas japonesas, encontrado que un 81,2\% de los participantes refirieron haber sufrido al menos un episodio de acoso en los últimos 6 meses y éste se asoció, en ambos géneros, con la aparición de cuadros depresivos $(\mathrm{p}<0,05)$.

NazanBilgel $^{12}$ en 2006 también encuentra una relación significativa entre el acoso laboral y la depresión con una OR 1, 7 (IC 95\% 1,1 7 - 2,46) p<0.005.

El estudio prospectivo de Georges Brousse ${ }^{11}$ en 2008 puso de manifiesto una prevalencia de síntomas depresivos entre pacientes víctimas del acoso laboral de un 52\% de, no produciéndose cambios a los 12 meses. Los resultados también mostraron una disminución significativa de los síntomas depresivos en los paciente que dejaron de trabajar $(\mathrm{p}<0,01)$. 
En la Tabla 5 se muestran los artículos analizados, dirigidos a mostrar una posible relación entre acoso y síntomas de depresión.

Tabla 5. Estudios que analizan la relación acoso - depresión

\begin{tabular}{|c|c|c|c|c|c|}
\hline Autor & Año & Muestra & Diseño & Medida de asociación & $\begin{array}{c}\text { Nivel de } \\
\text { evidencia }\end{array}$ \\
\hline Rugulies R & 2012 & 5.701 & Prospectivo & $\begin{array}{l}\text { OR: } 6,29 \text { (IC } 2,25-15,68) \text { expuestos } \\
\text { ocasional; OR de } 20.96(5,80-75,80) \\
\text { expuestos frecuente }\end{array}$ & $2-$ \\
\hline Taniguchi $\mathrm{T}$ & 2011 & 897 & Transversal & $\mathrm{X} 2 \exp /$ no exp $\mathrm{p}<0,05$ & 3 \\
\hline D. Yildirim & 2009 & 286 & Transversal & ANOVA $(\Omega=0,54 ; \mathrm{F}=56,61 ; \mathrm{P}<0,00)$ & 3 \\
\hline Neidhammer I & 2006 & 7.694 & Transversal & $\begin{array}{l}\mathrm{X}^{2} \text { test } \mathrm{P}<.001 \text {. OR } 8.00 \text { (IC } 95 \% 6,06-10,56 \text { ) } \\
\text { en hombres y una OR de } 8,44 \text { (IC } 95 \% 6,84 \\
-10,41 \text { ) }\end{array}$ & 3 \\
\hline Aytac Serpil & 2011 & 2.161 & Transversal & Mann-Whitney U teste, $\mathrm{P}=001$ & 3 \\
\hline Jiro Takaki & 2010 & 2.634 & Transversal & $\mathrm{p}<0,05$ & 3 \\
\hline Georges Brousse & 2008 & 48 & Prospectivo & T-Student; $\mathrm{p}<0,01$ & $2-$ \\
\hline Nazan Bilgel & 2006 & 1.200 & Transversal & OR 1,70 (IC 95\% $1,17-2,46$ ) $\mathrm{P}<0,005$ & 3 \\
\hline
\end{tabular}

En relación a los factores asociados con la vulnerabilidad al acoso laboral sólo encontramos resultados en el estudio realizado por Georges Brousse ${ }^{11}$ en el que mediante un diseño prospectivo siguió a 48 pacientes víctimas de acoso laboral durante 12 meses, este estudio pone de manifiesto que en su mayoría estos pacientes presentaban rasgos de personalidad neurótica, que según el autor se caracteriza por una excesiva sensibilidad emocional para afrontar situaciones adversas.

Con respecto a la estimación de la prevalencia de acoso laboral, las cifras que se obtienen en los diferentes estudios presentan una gran variabilidad, oscilando entre un $8,78 \%$ dado por Neidhammer en población francesa y un $81,2 \%$ dado por Jiro Takaki en población japonesa (Tabla 6).

Tabla 6. Prevalencia de acoso laboral

\begin{tabular}{lrlllc}
\hline \multicolumn{1}{c}{ Autor } & Muestra & \multicolumn{1}{c}{ Diseño } & Población & Prev. & Nivel de evidencia \\
\hline Rugulies R & 5.701 & Prospectivo & Danesa & $11,9 \%$ & 2 - \\
Aytac Serpil & 2.161 & Transversal & Turca & $44,8 \%$ & 3 \\
D. Yildirim & 286 & Transversal & Turca & $21,0 \%$ & 3 \\
Nazan Bilgel & 1.200 & Transversal & Turca & $55,0 \%$ & 3 \\
Taniguchi T & 897 & Transversal & Japonesa & $40,0 \%$ & 3 \\
Jiro Takaki & 2.634 & Transversal & Japonesa & $81,2 \%$ & 3 \\
Georges Brousse & 63 & Prospectivo & Francesa & $75,0 \%$ & $2-$ \\
Neidhammer I & 7.694 & Transversal & Francesa & $8,78 \%$ & 3 \\
\hline
\end{tabular}

No se pudo abordar el objetivo referido a la relación entre el acoso y el absentismo laboral dado que no encontramos publicaciones que abordaran este aspecto.

En lo referente a las variables género y actividad económica como condiciones de susceptibilidad; D. Yildirim ${ }^{14}$ y Rugulies R. $^{16}$ en sus estudios incluyen población exclusivamente femenina en colectivos de enfermería y cuidadoras de ancianos respectivamente.

Nazan Bilgel ${ }^{12}$ y Jiro Takaki ${ }^{10}$ no encuentran diferencia significativa en cuanto al acoso laboral y el género de la víctima. 
Los resultados de Nazan Bilgel muestran una frecuencia mayor de situaciones de acoso en personal con cargos de responsabilidad (60\%) en su población encontró una frecuencia de situaciones de acoso variable en función de la ocupación o sector de actividad: $75 \%$ en personal sanitario (excepto médicos), $64 \%$ en personal administrativo, $56 \%$ en personal policial, $56 \%$ en médicos y $39 \%$ en profesores.

Georges Brousse ${ }^{11}$ en el seguimiento de su cohorte de 48 pacientes víctimas de acoso laboral encontró que un $75 \%$ eran mujeres, un $70 \%$ trabajaban en el sector privado siendo la ocupación más frecuente la de personal administrativo (29\%), seguida de obreros y trabajadores no calificados (27\%) y dependientes (17\%).

Serpil Aytac ${ }^{9}$ en su muestra de 1.708 trabajadores de los cuales 1.334 estuvieron expuestos al acoso laboral encontró que el $68,7 \%$ eran mujeres, siendo el sector Sanitario con 51,3\% donde se encontró con mayor frecuencia casos de acoso; seguido del sector de seguridad (38,8\%), educación (36,8\%), servicios (26,6\%) e Industria (24,3\%).

Neidhammer I. ${ }^{13}$ encuentra una variación de acoso según género de 8,78\% en varones y $10,70 \%$ en mujeres.

La variación en la prevalencia del acoso laboral según género y actividad figura en la Tabla 7.

Tabla 7. El género y la actividad económica como condicionantes

\begin{tabular}{|c|c|c|c|c|c|}
\hline Autor & Muestra & Diseño & Genero & Sector Económico & $\begin{array}{c}\text { Nivel de } \\
\text { evidencia }\end{array}$ \\
\hline Georges Brousse & 63 & Prospectivo & $\begin{array}{l}\text { M: } 75 \% \\
\text { H: } 25 \%\end{array}$ & $\begin{array}{l}29 \% \text { Administrativos, } \\
27 \% \text { Obreros y no cualificados, } \\
17 \% \text { dependientes. }\end{array}$ & $2-$ \\
\hline Aytac Serpil & 2.161 & Transversal & $\begin{array}{l}\text { M: } 68,7 \% \\
\text { H: } 31,3 \%\end{array}$ & $\begin{array}{l}\text { 51,3\% Sanidad, } \\
38,8 \% \text { Seguridad, } \\
\text { 36,8\% Servicios, } \\
24,3 \% \text { Industria }\end{array}$ & 3 \\
\hline Neidhammer I & 7.694 & Transversal & $\begin{array}{l}\text { Prevalencia } \\
\text { acoso: } \\
\text { M: } 10,7 \% \\
\text { H: } 8,78 \%\end{array}$ & - & 3 \\
\hline Jiro Takaki & 2.634 & Transversal & No diferencia & - & 3 \\
\hline D. Yildrim & 286 & Transversal & - & - & 3 \\
\hline Rugulies R. & 5.701 & Prospectivo & - & - & $2-$ \\
\hline Nazan Bilgel & 1.200 & Transversal & No diferencia & $\begin{array}{l}75 \% \text { sanidad, } \\
64 \% \text { administrativos, } \\
56 \% \text { policías } \\
56 \% \text { médicos, } \\
39 \% \text { profesores. }\end{array}$ & 3 \\
\hline
\end{tabular}

\section{CONCLUSIONES Y DISCUSIÓN}

La presente revisión sistemática nos muestra una escasa producción científica sobre la relación entre acoso laboral y depresión, cabe resaltar un claro predominio del diseños transversales que no permiten demostrar una relación de causalidad.

La revisión nos muestra que la prevalencia acerca de la percepción de ser víctima de acoso laboral varía sustancialmente considerando la población y el país de estudio. En Europa alrededor de 1 cada 20 trabajadores (5\%) manifiestan haber sido víctimas de acoso laboral en el último año ${ }^{2}$, por ejemplo en nuestro medio, encontramos alrededor del $2,2 \%$ en comparación con Francia con 9,5\%. En nuestra revisión se evidenciaron 
valores mucho más elevados que varían entre el $8,78 \%^{11}$, y el $81,2 \%{ }^{10}$ en poblaciones Francesas y Japonesas respectivamente.

En cuanto a las estrategias de acoso Serpil Aytac ${ }^{9}$ nos muestra que el abuso verbal (38\%) seguido del hostigamiento (30,3\%) son las formas de acoso más frecuentes, siendo el acoso sexual el referido con menor frecuencia (5\%), pero cuyas víctimas son en su mayoría mujeres con el $88,5 \%$; de forma similar Georges Brousse menciona que tácticas más utilizadas son la asignación de mayor carga laboral, la discriminación y el aislamiento laboral.

En nuestra revisión encontramos que en los estudios que incluyen población sanitaria dentro de la muestra como muestra, ésta mostró mayores niveles de prevalencia que el resto ${ }^{9,12}$ siendo enfermería la ocupación, con una tasas de prevalencias superiores.

En las poblaciones estudiadas, teniendo en cuenta las diferentes variables incluidas, ser mujer, tener estudios universitarios y ser un trabajador con cierta antigüedad en el puesto o tener mayor experiencia, caracterizan una mayor vulnerabilidad a sufrir situaciones de acoso. Estos resultados en lo que se refiere a género no es coincidente con dos revisiones anteriores en las que no se encontró que el género represente un factor de vulnerabilidad $^{10,12}$ específicamente, en el colectivo de enfermería en el que ser más joven y con menos experiencia incrementaba la probabilidad de sufrir situaciones de acoso ${ }^{14}$.

En cuanto a la vulnerabilidad asociada a características individuales, como la personalidad, solo George Brousse ${ }^{11}$ identificó que la mayoría de las víctimas de acoso presentaban una personalidad con rasgos de neurotocixidad que conlleva a una poca capacidad para afrontar situaciones adversas, sin embargo en vista del diseño de este estudio, no se puede concluir en la existencia de una relación de causalidad.

Centrándonos en el objetivo principal de la revisión, y en concordancia con la evidencia recogida hasta el momento, todos los estudios incluidos en esta revisión ${ }^{9-16}$, encuentran una asociación positiva y significativa entre el ser víctima de Acoso laboral y el manifestar alteraciones en su salud mental, estas alteraciones incluyen la depresión, la ansiedad y el estrés; cabe resaltar que la relación encontrada es unidireccional, ya que en algunos estudios se hace referencia a una relación ambidireccional, situación que Rugulies R. en su análisis prospectivo no encuentra; ya que menciona que el hecho de estar deprimido en su estudio no muestra una predisposición a ser acosado.

Jiro Takaki también establece una asociación significativa entre el acoso laboral y los trastornos del sueño ${ }^{10}$. No se encontraron estudios que respondan directamente en cuanto esta relación de acoso y depresión, repercuten en los niveles de absentismo laboral.

Es de suma importancia resaltar que éstos resultados demuestran que el acoso laboral no solamente es un problema desde el punto de vista organizacional, sino que conlleva consecuencias en la salud mental y física de los trabajadores que lo sufren.

\section{REFERENCIAS BIBLIOGRÁFICAS}

1. VII Encuesta Nacional de Condiciones de Trabajo del 2011 (VII ENCT). Instituto Nacional de Seguridad e Higiene en el Trabajo. Ministerio de Empleo y Seguridad Social. Gobierno de España. Publicado el 02 de Agosto del 2012.

2. Fifth European Working Conditions Survey Eurofound (2012). Publications Office of the European Union, Luxembourg.

3. Acoso Psicológico en el Trabajo. Observatorio Permanente de Riesgos Psicosociales. Secretaria de Salud laboral y Medio Ambiente UGT. Madrid, Noviembre 2008.

4. Nota Técnica Preventiva 476. El Instituto Nacional de Seguridad e Higiene en el Trabajo (INSHT),

5. Acoso Psicológico en el Trabajo. José Carlos Mingote Adán. Curso Superior de Medicina del Trabajo 201213. Escuela Nacional de Medicina del Trabajo. Instituto Nacional de Salud Carlos III.

6. El País - http://elpais.com/diario/2011/04/27/sociedad/1303855205_850215.html 
7. Organización Mundial de la Salud - www.who.int/countries/esp/es

8. Organización Mundial de la Salud http://www.who.int/mediacentre/news/notes/2012/mental_health_ day_20121009/es/index.html

9. Serpil Aytac. Workplace Violence: A Study of Turkish Workers. International Journal of Occupational Safety and Ergonomics(JOSE) 2011, Vol.17, $\mathrm{N}^{\circ}$ 4, 385-402.

10. Jiro Takaki . Workplace Bullying could Play Important Roles in the Relationships between Job Strain and Symptoms of Depression and Sleep Disturbance. J OccupHealth 2010; 52: 367-374.

11. Georges Brousse. Psychopathological features of a patient population of targets of workplace bullying. Occupational Medicine 2008;58:122-128.

12. Nazan Bilgel. Bullying in Turkish white-collar workers.Occupational Medicine 2006; 56:226-231.

13. Niedhammer Isabelle, Association between workplace bullying and depressive symptoms in the French working population. Journal of PsychosomaticResearch. 2006; 61; 251-259.

14. D. Yilirim. Bullying among nurses and its effects. International NursingReview. 2009 Dec; 56(4):504-1.

15. Taniguchi T. Associations between workplace bullying, harassment, and stress reactions of professional caregivers at welfare facilities for the elderly in Japan. SangyoEiseigakuZasshi. 2012;54(1):1-9.

16. Rugulies R, Bullying at work and onset of a major depressive episode among Danish female eldercare workers. ScandinavianJurnal of Work, Environment\&Health 2012; 38(3): 218-227.

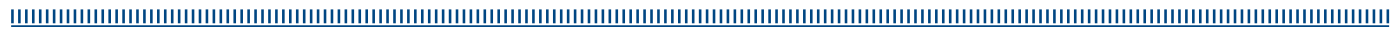

